\title{
Luvas antivibratórias: qual a evidência científica?
}

rpso.pt/luvas-antivibratorias-qual-a-evidencia-cientifica/

Santos M, Almeida A, Lopes C, Oliveira T. Luvas antivibratórias: qual a evidência científica? Revista Portuguesa de Saúde Ocupacional on line. 2020, volume 9, 1-16. DOI: 10.31252/RPSO.01.05.2020

\section{ANTIVIBRATIONS GLOVES: WHAT IS THE SCIENTIFIC EVIDENCE?}

\section{TIPO DE ARTIGO: Scoping Review}

\author{
Autores: Santos M(1), Almeida A(2), Lopes C(3), Oliveira T(4).
}

\section{RESUMO}

\section{Introdução/ enquadramento/ objetivos}

O uso de luvas antivibratórias não é tão frequente quanto outros equipamentos individuais de proteção, pelo que se torna uma área sobre a qual os profissionais a exercer nas equipas de Saúde Ocupacional (e até empregadores e trabalhadores) têm menor contato e experiência. Para além disso, quando se começa a pesquisar o tema, facilmente se percebe que existem dados contraditórios publicados, pelo que surgem muitas dúvidas sobre o benefício ou não do seu uso. Esta revisão pretende resumir o que de mais recente e pertinente se publicou sobre este tema.

\section{Metodologia}

Trata-se de uma Scoping Review, iniciada através de uma pesquisa realizada em dezembro de 2019 nas bases de dados "CINALH plus with full text, Medline with full text, Database of Abstracts of Reviews of Effects, Cochrane Central Register of Controlled Trials, Cochrane Database of Systematic Reviews, Cochrane Methodology Register, Nursing and Allied Health Collection: comprehensive, MedicLatina, SCOPUS e RCAAP”.

\section{Conteúdo}

Para que uma luva seja considerada antivibratória deverá cumprir com os critérios definidos pela Organização Internacional de Estandardização.

A sensibilidade aos danos associados às vibrações apresenta alguma variabilidade entre indivíduos.

As vibrações podem causar alterações vasculares, neurológicas e músculo-esqueléticas; no seu global, a situação designada por síndroma de vibração mão-braço (Hand Arm Vibration Syndrome); esta torna-se mais frequente quer com o aumento do tempo de exposição, quer com a intensidade.

Para além da proteção direta que possa existir em relação às vibrações, ao manter as mãos secas e quentes os danos vibratórios em si podem ficar atenuados indiretamente. Independentemente da questão vibratória, o trabalhador fica mais protegido também em relação a cortes, queimaduras, agentes químicos e biológicos. 
O maior risco poderá ser então dar ao empregador e funcionário a sensação de proteção, quando esta não for real. Para além disso, alguns investigadores consideram que certas medidas de proteção coletiva são mais eficazes que as luvas.

\section{Conclusões}

Os dados publicados são contraditórios e os estudos utilizaram geralmente amostras pequenas, pelo que se torna complicado generalizar os resultados com segurança e robustez científica. Para além disso, facilmente se percebe que a protecção, ausência desta ou até potenciação das vibrações dependerá de inúmeras variáveis, nomeadamente instrumento de trabalho, tipo de vibrações, modelo/material e espessura da luva na região palmar e dedos, bem como força de preensão exercida pelo trabalhador e as suas dimensões antropométricas. Trata-se pois de um equipamento de proteção que, de forma alguma, se pode considerar como uma maisvalia adquirida, para qualquer tarefa ou trabalhador.

PALAVRAS/ EXPRESSÕES- CHAVE: vibrações, luvas anti vibratórias, saúde ocupacional e medicina do trabalho.

\section{ABSTRACT}

\section{Introduction / background / objectives}

The use of anti-vibration gloves is not as frequent as other individual protective equipment, so it becomes an area in which the professionals that exercise in the Occupational Health teams (and even employers and workers) have less contact and experience. In addition, when starting to research the topic, it is easy to see that there are contradictory data published, so there are many doubts about the benefit or not on its use. This review intends to summarize the most recent and pertinent articles published on this topic.

\section{Methodology}

This is an Integrative Bibliographic Review, initiated through a research carried out in December 2019 in the databases "CINALH plus with full text, Medline with full text, Database of Abstracts of Reviews of Effects, Cochrane Central Register of Controlled Trials, Cochrane Database of Systematic Reviews, Cochrane Methodology Register, Nursing and Allied Health Collection: comprehensive, MedicLatina, SCOPUS and RCAAP".

\section{Content}

For a glove be considered anti-vibration, it must comply with the criteria defined by the International Standardization Organization.

The sensitivity to damage associated with vibrations has some variability between individuals.

Vibrations can cause vascular, neurological and musculoskeletal changes; overall, the situation referred to as hand-arm vibration syndrome; this becomes more frequent with increasing exposure time and intensity.

In addition to the direct protection that may exist in relation to vibrations, by keeping the hands dry and warm, vibratory damage can be indirectly attenuated. In addition, regardless of the vibration issue, the worker is also more protected from cuts, burns, chemical and biological agents.

The greatest risk may then be to give the employer and employee a sense of protection when it is not real. In addition, some researchers believe that certain collective protection measures are more effective than gloves.

\section{Conclusions}

The published data are contradictory and the studies generally used small samples, so it is difficult to generalize the results with safety. In addition, it is easy to see that the protection, absence of it or even potentiation of vibrations will depend on numerous variables, namely working instrument, type of vibrations, 
model/ material and glove thickness in the palm and fingers, as well as grip strength exercised by the worker and its anthropometric dimensions. It is therefore a protective equipment that, in no way, can be considered as an added value, for any task or worker.

KEY-WORDS / EXPRESSIONS: vibrations, anti-vibration gloves, occupational health and occupational medicine.

\section{INTRODUÇÃO}

O uso de luvas antivibratórias não é tão frequente quanto outros equipamentos individuais de proteção, pelo que se torna uma área sobre a qual os profissionais a exercer nas equipas de Saúde Ocupacional (e até empregadores e trabalhadores) têm pouco contato e experiência. Para além disso, quando se começa a pesquisar o tema, facilmente se percebe que existem dados contraditórios publicados, pelo que surgem muitas dúvidas sobre o benefício ou não no seu uso. Esta revisão pretende resumir o que de mais recente e pertinente se publicou sobre este tema.

\section{METODOLOGIA}

Em função da metodologia PICo, foram considerados:

-P (population): trabalhadores sujeitos a vibrações e que possam ponderar usar luvas anti vibratórias.

-I (interest): consequências do uso de luvas antivibratórias

-C (context): saúde ocupacional nas empresas com postos de trabalho com vibrações e com disponibilização de uso de luvas antivibratórias.

Pergunta protocolar: as luvas anti vibratórias oferecem proteção eficaz?

Foi realizada uma pesquisa em dezembro de 2019 nas bases de dados "CINALH plus with full text, Medline with full text, Database of Abstracts of Reviews of Effects, Cochrane Central Register of Controlled Trials, Cochrane Database of Systematic Reviews, Cochrane Methodology Register, Nursing and Allied Health Collection: comprehensive, MedicLatina, Academic Search Ultimate, Science Direct, Web of Science, SCOPUS e RCAAP”.

No quadro 1 podem ser consultadas as expressões/ palavras-chave utilizadas nas bases de dados e restantes dados associados à seleção dos documentos.

\section{CONTEÚDO}

\section{Vibrações: setores profissionais, estatísticas de alguns países e legislação associada}

Estimou-se que cerca de 17\% dos trabalhadores europeus estão expostos a vibrações, pelo menos, em metade do seu horário. Por sua vez, em Portugal, quantificou-se que cerca de $33 \%$ dos funcionários estavam expostos, no mínimo, num quarto do seu dia de trabalho (1).

Outros documentos publicaram que 1,7 a 3,6\% dos trabalhadores europeus e americanos estão expostos a vibrações com capacidade de causar dano. Acredita-se que cerca de 30 a 90\% dos expostos desenvolverão lesões significativas. Por exemplo, a nível de $2,5 \mathrm{~m} / \mathrm{s}^{2}$, por 12 anos, pensa-se que tal implicará cerca de $10 \%$ de probabilidade de apresentar a síndroma de vibração mão-braço (HAVS- hand-arm vibration syndrome) (2).

Outros investigadores, por sua vez, estimaram que, nos EUA, entre 8 a 10 milhões de trabalhadores estão expostos a vibrações; no Reino Unido acredita-se que esse valor será na ordem de 1 milhão (considerando sete horas por dia); a maioria pertence ao setor da construção e outras profissões com tarefas manuais. No 
Canadá calculou-se que cerca de quarenta grupos profissionais estão expostos a vibrações. Por exemplo, entre mineiros, um estudo quantificou uma prevalência de cerca de 50\% de HAVS e 25\% apresentava outras alterações associadas às vibrações (3).

Entre os diversos setores profissionais, destacam-se então a construção, a indústria transformadora e extrativa, agricultura e pesca, setor da eletricidade/ gás, transportes e armazenagem. As fontes de vibração podem ser algumas máquinas e ferramentas manuais (1).

Para atenuar a questão existem diversas normas que pretendem regulamentar limites máximos de exposição; contudo, nem sempre os pontos de corte são adequados e/ ou tal é cumprido (3). Após a aplicação das medidas de proteção coletiva, se ainda se exceder o valor máximo permitido, torna-se necessário o uso de equipamentos de proteção individual, como as luvas, ainda que pouco estudadas de forma robusta na literatura internacional (1).

Em Portugal é o Decreto-Lei 46/2006 que transpõe a Diretiva Europeia 2002/44/CE; assim, o valor limite de exposição é de 5 e o valor de ação de exposição é $2,5 \mathrm{~m} / \mathrm{s}^{2}(1)$ (2) , por oito horas. Outras recomendações assinalam para vibrações mão-braço até 12, 8,6 e $4 \mathrm{~m} / \mathrm{s}^{2}$ para tempos de exposição de menos que uma hora, de uma a duas, de duas a quatro e de quatro a oito horas, respetivamente (4). Contudo, salienta-se que há uma grande variabilidade na suscetibilidade entre indivíduos (1).

Para que uma luva seja considerada anti vibratória deverá cumprir com os critérios definidos pela Organização Internacional de Estandardização (ISO) 1081, de 2013 (5). A generalidade das luvas é feita com materiais como gel, espuma, borracha e/ ou equivalentes; por vezes até com bolsas de ar inseridas (6) (7).

Os empregadores não devem assumir que qualquer modelo de luva tem capacidade para atenuar as vibrações de qualquer equipamento, em qualquer funcionário (6).

Parte dos trabalhadores não considera que a exposição a vibrações possa levar a problemas médicos, uma vez que elas não são visíveis e os sintomas/ diagnósticos não surgem imediatamente após a exposição. Logo, parte destes não usam as luvas antivibratórias (4).

\section{Consequências médicas associadas às Vibrações}

Como já se mencionou, a sensibilidade aos danos associados às vibrações apresenta variabilidade entre indivíduos (4).

As vibrações podem causar alterações vasculares, neurológicas (2) (5) e músculoesqueléticas (1) (2) (3) (5) (8); no seu global, a situação designa-se por síndroma de vibração mãobraço (2) (3) (8) (9). O HAVS torna-se mais frequente quer com o aumento do tempo de exposição, quer com a intensidade (4). Outros artigos realçam que esta patologia depende não só do nível de vibrações e da duração da exposição diária e ao longo dos anos; mas também da temperatura, método de trabalho, postura do funcionário, forma de preensão e sensibilidade individual (2).

As alterações vasculares associadas às vibrações justificam-se fisiopatologicamente pela interrupção do fluxo sanguíneo para as extremidades, com destaque para os dedos, manifestada pela alteração de cor (esbranquiçada) (1) (2) (5) (8), eventualmente potenciada pelo frio-fenómeno de Raynaud ou síndroma dos dedos brancos induzido pelo frio (VWF-vibration white fingers) (1) (2) (3) (7) (10). Após o tom branco poderá surgir a cor azulada (representativa de cianose), devido à diminuição da concentração de oxigénio; por sua vez, durante a recuperação, eventualmente devido ao calor e/ ou massagem, o fluxo sanguíneo aumenta e poderá surgir um tom rosado/ avermelhado. A longo prazo alguns investigadores publicaram que poderão surgir alterações tróficas, como úlceras ou até gangrena (1) (2).

Em contexto neurológico, podem surgir parestesias que, se intensas, poderão interferir com o desempenho laboral e/ ou tarefas pessoais (1). 
O período médio de latência em relação aos sintomas vasculares é de 19,7 anos e, para as questões neurológicas 19,1 anos; contudo, a prevalência destas últimas é geralmente o dobro (1) (2).

No setor da construção, por exemplo, cerca de 10\% apresenta sintomas vasculares e 14\% neurológicos (2). As alterações na sensibilidade e destreza manual (5) (8) (7), bem como dor e diminuição da força (8) (7), por sua vez, podem potenciar a ocorrência de acidentes de trabalho (1) (2).

Alguns investigadores defendem a possibilidade de a exposição a vibrações ter a capacidade de potenciar a hipoacusia, devido à vasoconstrição nas estruturas do ouvido interno, secundária às vibrações (1).

As vibrações podem potenciar a ocorrência de síndroma do túnel cárpico (devido à compressão do nervo mediano no punho), reconhecida como doença profissional (2). Poderá também ocorrer diminuição da força (2) (5), outras tendinopatias e, eventualmente, formação de quistos (controverso entre investigadores) (2).

\section{Vantagens que as Luvas Antivibratórias conseguem proporcionar}

O uso destas luvas tem como objetivo diminuir a transmissão de vibrações ao sistema mão-braço (11).

Alguns investigadores especificaram que as luvas por eles avaliadas conseguem atenuar as vibrações se estas forem superiores a $100 \mathrm{Hzs}$; no entanto, pode ocorrer o inverso nas restantes frequências (1). Curiosamente, existem também estudos publicados que concluíram que os modelos avaliados atenuaram as vibrações para todos os dedos em $3 \%$ para frequências inferiores a $80 \mathrm{Hzs}$, mas aumentando as mesmas entre os 80 e 400 Hzs; aqui os modelos com gel incorporado demonstraram-se mais eficazes (12). Outros ainda defendem que a generalidade dos modelos é efetiva a atenuar vibrações com frequências elevadas (7). Há também quem tenha concluído que a atenuação das vibrações foi na ordem dos $86 \%$, através do uso dos modelos que analisaram (3). Alguns defendem que a maioria das luvas não apresenta grande proteção antivibratória para frequências inferiores a $25 \mathrm{Hzs}$; aliás, até as pode potenciar (6). Enquanto que alguns estudos afirmam que as luvas antivibratórias são eficazes (5) (12) (13) (14), outros discordam, ou seja, até consideram que em algumas circunstâncias tal pode ficar potenciado (5). Ou seja, não é possível comparar estudos que utilizaram diferentes modelos de luvas, trabalhadores e equipamentos de trabalho.

Para além da proteção direta que possa existir em relação às vibrações, ao manter as mãos secas e quentes (9), os danos vibratórios podem ficar atenuados indiretamente (1) (2). Contudo, independentemente da questão vibratória, o trabalhador fica mais protegido também em relação a cortes, queimaduras, agentes químicos e biológicos (5) (9).

\section{Desvantagens associadas às Luvas Anti vibratórias}

Não há evidência científica robusta que comprove a eficácia das luvas antivibratórias; outros consideram que esta não é constante ou confiável (8) (9). O maior risco poderá ser então dar ao empregador e funcionário a sensação de proteção, quando esta não for real (5). Para além disso, alguns investigadores consideram que certas medidas de proteção coletiva são mais eficazes que as luvas (9).

Numa investigação de mestrado nacional concluiu-se que o uso de luvas antivibratórias não altera de forma estatisticamente significativa a transmissão de vibrações ao sistema mão-braço; sendo que, por questões de conforto, os trabalhadores assinalaram que preferiam não usar esse equipamento de proteção (1). Elas podem alterar o desempenho (através da sensibilidade, desempenho, destreza, força de preensão e amplitude dos movimentos) e, uma vez que tal pode ser percecionado pelo trabalhador, surge por vezes alguma resistência à sua utilização (2) (9). As luvas antivibratórias são geralmente mais grossas, o que aumenta o esforço para agarrar os instrumentos de trabalho, potenciando a fadiga muscular (e o risco de síndroma do túnel cárpico), prejudicando a destreza manual (9) (12) (15) e/ ou potenciando a sudorese (4). Para além disso, como já se mencionou, existem publicações que concluíram que, em algumas circunstâncias, as vibrações podem até ficar potenciadas (1) (2) (5). 
Num projeto onde se utilizou a eletromiografia de superfície, de forma a registar a atividade muscular (do flexor radial do carpo, flexor superficial dos dedos, extensor ulnar do carpo e o extensor dos dedos), em funcionários a utilizar diversos equipamentos, verificou-se que a utilização de luvas antivibratórias não aumentou o esforço nos músculos avaliados em repouso mas, utilizando as ferramentas, tal ocorreu na ordem $\operatorname{dos} 12$ a $34 \%(11)$.

Está publicado que as luvas de algodão aumentam o esforço muscular e as de nylon fazem o inverso; outros, por sua vez, consideram que não existem diferenças estatisticamente significativas ou então que apenas as luvas feitas de material mais endurecido é que potenciam o esforço (quer dos músculos extensores, quer flexores) (1).

\section{Fatores que têm capacidade de interferir com o efeito das Luvas Antivibratórias}

São inúmeros os fatores assinalados na literatura como capazes de modular a eficácia das luvas; podem ser realçados os seguintes:

-tipo de instrumento de trabalho (1) (10) (16)

-caraterísticas das vibrações (5) (7) (8) (10) (16) (frequência, incidência e ou direção)

$-\operatorname{tarefa}(1)(5)$

-material da luva (1) (2) (6) (7) (8) (16) (17) (18) (19), propriedades viscoelásticas (2)

-espessura da luva na região palmar e dedos (2) (19)

-ajuste da luva (2)

-posição do braço (1) (2) e dedos (10) (um menor angulo no cotovelo potencia a transmissão das vibrações, por exemplo) ${ }^{(2)}$

-tamanho da mão (2) (7) (18), outras condições antropométricas do trabalhador (5) e resposta global do sistema mão-dedos (18) (19)

-força de preensão (6) (7) (8) (20) e

-ajuste da luva (8).

Por vezes verifica-se que a mesma luva tem eficácia antivibratória num grupo de trabalhadores e, noutro, o efeito oposto (2) (9). A avaliação da eficácia depende muito das caraterísticas dos indivíduos da amostra de estudo, pelo que os resultados, entre amostras diferentes, poderão ser discordantes. Para além disso, a proteção não é homogénea entre diferentes direções, ou seja, o benefício proporcionado para a região palmar é diferente do dos dedos (9).

Os modelos que mais protegem, geralmente são os que diminuem mais o desempenho (2).

A transmissão das vibrações pode ser atenuada ou potenciada de acordo com o material da luva. As luvas antivibratórias atenuam a destreza manual de forma mais intensa que os modelos de luvas generalistas avaliados (17).

A maioria dos trabalhadores de uma investigação referiu que se sentia mais confortável em não usar este equipamento, uma vez que tal exigia mais força para agarrar no instrumento de trabalho e aumentava a sudorese (4). Tal poderá ser potencialmente atenuado com o uso de luvas com dedos cortados, mantendo uma proteção para as vibrações semelhante (4) (17); ou seja, 90\% dos funcionários avaliados afirmou sentirse confortável com este tipo de luva (4), ainda que não cumpram com todos os critérios definidos pela ISO10819 (17). 
As caraterísticas dos materiais usados nestes EPIs são moduladas pela temperatura, humidade, fotoirradiação e pressão de contato, que envelhecem o produto e atenuam a sua proteção antivibratória, mesmo que não usadas, ainda embaladas, sem qualquer uso prévio; mas sobretudo quando utilizadas por períodos consideráveis. Contudo, o embalamento fazendo uso de vácuo pode atenuar o problema (21).

Para frequências entre os 20 e os 350 Hzs, houve diminuição das vibrações a nível palmar, mas aumento em contexto digital. Ou o aumento da espessura da luva pode diminuir a vibração na palma mas aumentar nos dedos (19). Em princípio, as luvas fornecem o efeito de almofada/ amortecimento entre o instrumento de trabalho e a mão. Genericamente, este equipamento de proteção é mais eficaz para a palma que os dedos. Em teoria, diminuindo-se a rigidez e aumentando o amortecimento, o isolamento da luva fica potenciado. Tal pode ser conseguido usando materiais mais suaves ou aumentando a espessura do lado palmar. Contudo, estas condições poderão não ser as mais adequadas para segurar adequadamente os instrumentos de trabalho e/ ou pode ser necessário um esforço/ força superiores. Daí que ao aumentar a potência da proteção antivibratória, geralmente diminui-se o desempenho e a segurança (em relação a outros parâmetros) (9). A atenuação proporcionada para os dedos não tem de ser equivalente à dada às restantes partes da mão; até porque a espessura do material pode atingir os $55 \%$ da palma, por exemplo. Reduzindo a força de preensão, também se atenua a transmissão das vibrações (12). Ou seja, materiais mais espessos e suaves serão mais eficazes a nível de proteção antivibratória; contudo, maior suavidade poderá potenciar a sinistralidade e maior espessura potencia a força de preensão (6), como já se referiu.

Alguns estudos concluíram que as luvas avaliadas pouco atenuavam a transmissão de vibrações, sendo até que, em alguns casos, elas ampliavam tal em cerca de 10\% (sobretudo as de neopreno), ainda que, com alguns instrumentos de trabalho, até essa se demonstrou eficaz (10).

Avaliando vários modelos de luvas percebeu-se que apenas aqueles com bolsa de ar e hibridas é que conseguiam proporcionar alguma atenuação das vibrações para os dedos; aliás, quase todas ampliavam as vibrações para o dedo médio (22).

Logo, uma investigação que pretenda avaliar a eficácia de um modelo de luvas deve incluir vários funcionários com caraterísticas diversas de mãos e com a aplicação de diferentes forças (20).

\section{Normas para a uniformização da investigação da eficácia das luvas}

Existe alguma falta de uniformidade em relação aos métodos para quantificar a proteção das luvas, situação essa que algumas normas tentam atenuar (2). Para que uma luva seja considerada antivibratória deve passar alguns testes, determinados por algumas normas ISO (International Organization for Standartization) (10). É o caso da ISO 10819 (5) (6) (8) (9) (12) de 2013; bem como a ISO 5349-1 de 2001 (6) (9); ISO 13753 de 2008 e a ISO 10068 de 1998 (9), por exemplo. A primeira aqui mencionada especifica a espessura máxima na palma e dedos (6).

\section{DISCUSSÃO/ CONCLUSÃo}

Os dados publicados são contraditórios e os estudos utilizaram geralmente amostras pequenas, pelo que se torna complicado generalizar os resultados com segurança e robustez científica. Para além disso, facilmente se percebe que a protecção, ausência desta ou até potenciação das vibrações dependerá de inúmeras variáveis, nomeadamente instrumento de trabalho, tipo de vibrações, modelo/ material e espessura da luva na região palmar e dedos, bem como força de preensão exercida pelo trabalhador e as suas dimensões antropométricas. Trata-se pois de um equipamento de protecção que, de forma alguma, se pode considerar como uma maisvalia uniforme, para qualquer tarefa ou trabalhador.

\section{CONFLITOS DE INTERESSE, QUESTÕES ÉTICAS E/OU LEGAIS}


Nada a declarar.

\section{AGRADECIMENTOS}

Nada a declarar.

\section{BIBLIOGRAFIA}

1. Milho R. O esforço, o conforto e a vibração no sistema mão-braço, associados à utilização de luvas anti vibratórias. Mestrado em Engenharia e Gestão Industrial. Faculdade de Ciências e Tecnologia, Universidade Nova de Lisboa. 2009, 1-186.

2. Messias R. A eficácia das luvas anti vibratórias no amortecimento das vibrações transmitidas ao punho. Mestrado em Engenharia e Gestão Industrial. Faculdade de Ciências e Tecnologia, Universidade Nova de Lisboa. 2014, 1-156.

3. Milosevic M, McConville K. Evaluation of Protective Gloves and working techniques for reducing hardarm vibration exposure in the workplace. Journal of Occupational Health. 2012, 54, 250-253.

4. Masaud N, Abdullah B. Evaluation of different type of glove using vibration hand tool. Jurnal Teknologi. 2015, 76(6), 13-18.

5. Budd B, House R. Examining the usefulness of ISSO 10819 anti-vibration glove certification. Annals of Work Exposures and Health. 2017, 61(2), 137-140. DOI: 10.1093/annweh/wxw018

6. Hewitt S, Dong R, McDowell T, Welcome D. The Efficacy of Anti-vibration gloves. Acoustics Australia. 2015, 44(1), 121-127. DOI: 10.1007/s40857-015-0040-5

7. Xu X, Riley D, Person M, Welcome D, Krajnak K, Wu J et al. Evaluation of anti-vibration effectiveness of glove materials using animal model. Bio-Medical Materials and Engineering. 2011, 21, 193-211. DOI: 10.3233/BME-2011-0669

8. Krajnak K, Waugh S, Johnson C, Miller R, Welcome D, Xu X et al. Antivibration gloves: effects on cardiovascular and sensorineural function, an animal model. Journal of Toxicology and Environmental Health. 2015, 78(9), 571-582. DOI: 10.1080/15287394.2015.1014079

9. Hewitt S, Dong R, Welcome D, McDowell T. Anti-vibration gloves? Annals of Occupacional Hygiene. 2015, 59(2), 127-141. DOI: 10.1093/annhyg/mevo89

10. Welcome D, Dong R, Xu X, Warren C, McDowell T. Tool-specific performance of vibration-reducing gloves for attenuating fingers- transmitted vibration. Occupational Ergonomy. 2016, 13(1), 23- 44. DOI: 10.3233/OER-160235

11. Roque B. O esforço no antebraço associado à vibração e à utilização de luvas anti vibratórias. Mestrado em Engenharia e Gestão Industrial. Faculdade de Ciências e Tecnologia, Universidade Nova de Lisboa. 2013, 1-141.

12. Welcome D, Dong R, Xu X, Warnen C, McDowell T. The effects on vibration- reducing gloves on finger vibration. International Journal of Industrial Ergonomics. 2014, 44(1), 45- 49. DOI:

10.1016/j.ergon.2013.10.003

13. Hermann T, Dobry M. Energy evaluation of protection effectiveness of anti-vibration gloves: International Journal of Occupational Safety and Ergonomics. 2017, 23(3), 4125-423. DOI: 10.1080/10803548.2016.1233673

14. Hermann T, Dobry M. Assessment of the effectiveness of antivibration gloves. A comparison of the conventional and energy method. Analysis and interpretation of results- part two. Vibrations in Physical Systems. 2017, 28, 1-8.

15. Cabeças J, Milho R. The efforts in the forearm during the use of antivibration gloves in simulated work tasks. International Journal of Industrial Ergonomics. 2011, 41(3), 289-297.

16. Kowalski P. Examining the Effectiveness of Anti-Vibration Gloves with a neural network. International Journal of Occupational Safety and Ergonomics. 2011, 17(3), 241-247. DOI: 10.1080/108003548.2011.11076891

17. Yao Y, Rakheja S, Gauvin C, Marcotte P, Hamouda K. Evaluation of effects of anti-vibration gloves on manual dexterity. Ergonomics. 2018, 61(11), 1530-1544. DOI: 10-1080/00140139.2018.1497208 
18. Rezali K, Griffin M. Transmission of vibration through gloves: effects of contact area. Ergonomics. 2017, 60(1), 60-81. DOI: 10. 1080/00140139.2016.1170210

19. Rezali K, Griffin M. Transmission of vibration through gloves: effects of material thickness. Ergonomics. 2016, 59(8), 1026-1037. DOI: 10. 1080/00140139.2015.1102334

20. Laszlo H, Griffing M. The transmission of vibration through gloves: effects of push force, vibration magnitude and inter-subject variability. Ergonomics. 2011, 54(5), 488-496. DOI: 10.1080/00140139.2011.562984

21. Shibata N. Effect of shelf aging on vibration transmissibility of anti-vibration gloves. Industrial Health. 2017, 55, 575- 579 .

22. Hamouda K, Rakheja S, Marcotte P, Dewangan K. Fingers vibration transmission performance of vibration reducing gloves. International Journal of Industrial Ergonomics. 2017, 62, 55-69.

Quadro 1- Metodologia utilizada na pesquisa da revisão bibliográfica

\begin{tabular}{|c|c|c|c|c|c|c|c|}
\hline $\begin{array}{l}\text { Bases de } \\
\text { dados }\end{array}$ & $\begin{array}{l}N^{\circ} \text { da } \\
\text { pesquisa }\end{array}$ & $\begin{array}{l}\text { Palavras/ } \\
\text { expressões- } \\
\text { chave }\end{array}$ & $\begin{array}{l}\mathrm{N}^{\circ} \text { de } \\
\text { artigos } \\
\text { obtidos }\end{array}$ & $\begin{array}{l}\text { Pesquisa } \\
\text { efetivada } \\
\text { ou não }\end{array}$ & $\begin{array}{l}\text { Artigos } \\
\text { selecionados } \\
\text { (nº na } \\
\text { pesquisa) }\end{array}$ & $\begin{array}{l}\text { Codificação } \\
\text { inicial }\end{array}$ & $\begin{array}{l}\text { Codificação } \\
\text { final }\end{array}$ \\
\hline \multirow{4}{*}{$\begin{array}{l}\text { RCAAP } \\
\text { (Repositório } \\
\text { Científico de } \\
\text { Acesso } \\
\text { Aberto em } \\
\text { Portugal) }\end{array}$} & 1 & $\begin{array}{l}\text { Luvas anti- } \\
\text { vibração }\end{array}$ & 2 & $\operatorname{sim}$ & $\begin{array}{l}1 \\
2\end{array}$ & $\begin{array}{l}1.1 \\
1.2\end{array}$ & $\begin{array}{l}11 \\
1\end{array}$ \\
\hline & 2 & $\begin{array}{l}\text { Luvas anti- } \\
\text { vibrações }\end{array}$ & 1 & $\operatorname{sim}$ & 1 & 2.1 & 2 \\
\hline & 3 & $\begin{array}{l}\text { Luvas anti } \\
\text { vibratórias }\end{array}$ & 3 & $\operatorname{sim}$ & $\begin{array}{l}1 \\
2\end{array}$ & $\begin{array}{l}=1.1 \\
=2.1\end{array}$ & \\
\hline & & & & & 3 & $=1.2$ & \\
\hline \multirow{11}{*}{$\begin{array}{l}\text { EBSCO } \\
\text { (CINALH, } \\
\text { Medline, } \\
\text { Database of } \\
\text { Abstracts } \\
\text { and } \\
\text { Reviews, } \\
\text { Central } \\
\text { Register of } \\
\text { Controlled } \\
\text { Trials, } \\
\text { Cochrane } \\
\text { Database of } \\
\text { Systematic } \\
\text { Reviews, } \\
\text { Nursing \& } \\
\text { Allied Health } \\
\text { Collection e } \\
\text { MedicLatina) }\end{array}$} & 4 & $\begin{array}{l}\text { Antivibration } \\
\text { aloves }\end{array}$ & 17 & $\operatorname{sim}$ & $\begin{array}{l}1 \\
2\end{array}$ & $\begin{array}{l}4.1 \\
42\end{array}$ & - \\
\hline & & & & & 6 & 4.3 & - \\
\hline & & & & & 7 & 4.4 & 13 \\
\hline & & & & & 8 & 4.5 & 5 \\
\hline & & & & & 9 & 4.6 & 18 \\
\hline & & & & & 10 & 4.7 & 19 \\
\hline & & & & & 11 & 4.8 & 9 \\
\hline & & & & & 12 & 4.9 & 8 \\
\hline & & & & & 14 & 4.10 & 3 \\
\hline & & & & & 15 & 4.11 & 20 \\
\hline & & & & & 16 & 4.12 & 16 \\
\hline
\end{tabular}




\section{(1)Mónica Santos}

Licenciada em Medicina; Especialista em Medicina Geral e Familiar; Mestre em Ciências do Desporto; Especialista em Medicina do Trabalho e Doutoranda em Segurança e Saúde Ocupacionais, na Faculdade de Engenharia da Universidade do Porto. Presentemente a exercer nas empresas Medicisforma, Servinecra, 
Securilabor e Medimarco; Diretora Clínica da empresa Quercia; Diretora da Revista Portuguesa de Saúde Ocupacional on line. Endereços para correspondência: Rua Agostinho Fernando Oliveira Guedes, 42, 4420oo9 Gondomar. E-mail: s_monica_santos@hotmail.com

\section{(2) Armando Almeida}

Enfermeiro Especialista em Enfermagem Comunitária, com Competência Acrescida em Enfermagem do Trabalho. Doutorado em Enfermagem; Mestre em Enfermagem Avançada; Pós-graduado em Supervisão Clínica e em Sistemas de Informação em Enfermagem; Professor Auxiliar Convidado na Universidade Católica Portuguesa, Instituto da Ciências da Saúde - Escola de Enfermagem (Porto) onde Coordena a PósGraduação em Enfermagem do Trabalho; Diretor Adjunto da Revista Portuguesa de Saúde Ocupacional on line.4420-009 Gondomar. E-mail: aalmeida@porto.ucp.pt

\section{(3)Catarina Lopes}

Licenciada em Enfermagem, desde 2010, pela Escola Superior de Saúde Vale do Ave. A exercer funções na área da Saúde Ocupacional desde 2011 como Enfermeira do trabalho autorizada pela Direção Geral de Saúde, tendo sido a responsável pela gestão do departamento de Saúde Ocupacional de uma empresa prestadora de serviços externos durante 7 anos. Atualmente acumula funções como Enfermeira de Saúde Ocupacional e exerce como Enfermeira Generalista na SNS24. Encontra-se a frequentar o curso Técnico Superior de Segurança do Trabalho. 4715-028. Braga. E-mail: catarinafflopes@gmail.com

\section{(4)Tiago Oliveira}

Licenciado em Enfermagem pela Universidade Católica Portuguesa. Frequenta o curso de Técnico Superior de Segurança no Trabalho. Atualmente exerce a tempo inteiro como Enfermeiro do Trabalho. No âmbito desportivo desenvolveu competências no exercício de funções de Coordenador Comercial na empresa Academia Fitness Center, assim como de Enfermeiro pelo clube de futebol União Desportiva Valonguense. 4435-718 Baguim do Monte. E-mail: tiago_sc16@hotmail.com 